\title{
CNDO/S-CI Calculations of some Carbonyl-containing Organic Luminophores with a Stilbene Subchromophore
}

\author{
G. Olbrich*, P. Nikolov**, F. Fratev $(\dagger)^{* *}$, and O. E. Polansky* \\ Z. Naturforsch. 40a, 859-863 (1985); received May 17, 1985 \\ For a series of carbonyl-containing compounds with a stilbene subchromophore the lowest ten \\ singlet and triplet states have been calculated with the aid of the CNDO/S-CI method. The \\ results were used to rationalize the relative ordering of the lowest-lying $S\left(\pi \pi^{*}\right), S\left(\mathrm{n} \pi^{*}\right)$, \\ $T\left(\pi \pi^{*}\right)$, and $T\left(\mathrm{n} \pi^{*}\right)$ electronic states. Using the calculated energy separation between the singlet \\ $\pi \pi^{*}$ and $n \pi^{*}$ states and between the singlet $\pi \pi^{*}$ and triplet $n \pi^{*}$ states the fluorescence ability of \\ the compounds studied is interpreted. The connection between the structure of the compounds \\ and their fluorescence is discussed. It is shown that an increase of the solvent polarity in some \\ cases may lead to an inversion of the singlet $n \pi^{*}$ and $\pi \pi^{*}$ levels and to an enhancement of the \\ fluorescence quantum yield.
}

\section{Introduction}

Recently, the results of investigations into the photophysical properties of a series of new organic luminophores with a stilbene skeleton - 3-phenylmethylene-1 $(3 \mathrm{H})$-isobenzofuranones (benzylidenephtalides, BPH's) and some of its derivatives were described [1-3]. The observed changes in the fluorescence quantum yield, $Q_{\mathrm{f}}$, with different substituents were explained with changes in the relative ordering of the lowest-lying $n \pi^{*}$ and $\pi \pi^{*}$ singlet states. This supposition is confirmed by the increase of $Q_{\mathrm{f}}[1-3]$ on raising the polarity of the solvent.

In this study we present the results of semiempirical calculations for the low-lying $\mathrm{n} \pi^{*}$ and $\pi \pi^{*}$ states of differently substituted carbonyl luminophores containing a stilbene subchromophore. The compounds studied comprised not only substituted BPH's but also related compounds with a stilbene skeleton (for the structures see Figure 1): I - BPH, II - 4-amino BPH, III - anhydride of BPH, IV - 3-phenylcumarine, V - 3-phenylisocumarine, VI - 1-oxo-3-phenylisoquinoline, VII 1-oxo-3-phenyl-4-hydroxyisoquinoline. The calculations were performed for the unsubstituted compounds I-VII (X $=\mathrm{H}$, see Fig. 1) together with the compounds derived from them by substitution with

* Max-Planck-Institut für Strahlenchemie, Stiftstraße 34-36, D-4330 Mülheim a. d. Ruhr.

** Institute of Organic Chemistry, Bulgarian Academy of Science, BG-1113 Sofia.

Reprint requests to Prof. O. E. Polansky, Max-PlanckInstitut für Strahlenchemie, Stiftstraße 34-36, 4330 Mülheim a. d. Ruhr.
$\mathrm{NH}_{2}$ and $\mathrm{CN}$ in the $4^{\prime}$ position of the phenyl group as indicated in Figure 1. For some of the compounds experimental data exist, while for others the calculations carried out should serve as a tentative assessment of their luminescence properties.

\section{Computational Method}

The computations in this study were performed by a modified CNDO/S-CI method [4] as used previously by Olbrich et al. [5] in a study of acenequinones. The INDO integral approximation was used to describe the splitting of singlet and triplet $\mathrm{n} \pi^{*}$ states; for the CI calculations singly excited configurations were used. For all compounds a planar geometry with standard bond lengths and bond angles was assumed.

\section{Results and Discussion}

The energies of the respective first five singlet and triplet excited states of the compounds I-VII with $\mathrm{X}=\mathrm{H}, \mathrm{NH}_{2}$, and $\mathrm{CN}$ have been calculated. In Fig. 1 the computed excitation energies to the lowest-lying singlet and triplet $\pi \pi^{*}$ and $n \pi^{*}$ states are shown; from now on we will denote these lowest-lying states by the symbols $S\left(\pi \pi^{*}\right), S\left(\mathrm{n} \pi^{*}\right)$, $T\left(\pi \pi^{*}\right)$, and $T\left(\mathrm{n} \pi^{*}\right)$. Figure 2 gives a comparison of the calculated excitation energies with the experimental absorption spectra of the unsubstituted compounds I, II, III, IV, V, and VII. With the exception of compounds II and V, where the first $\pi \pi^{*}$ states are somewhat too high, the agreement between the 


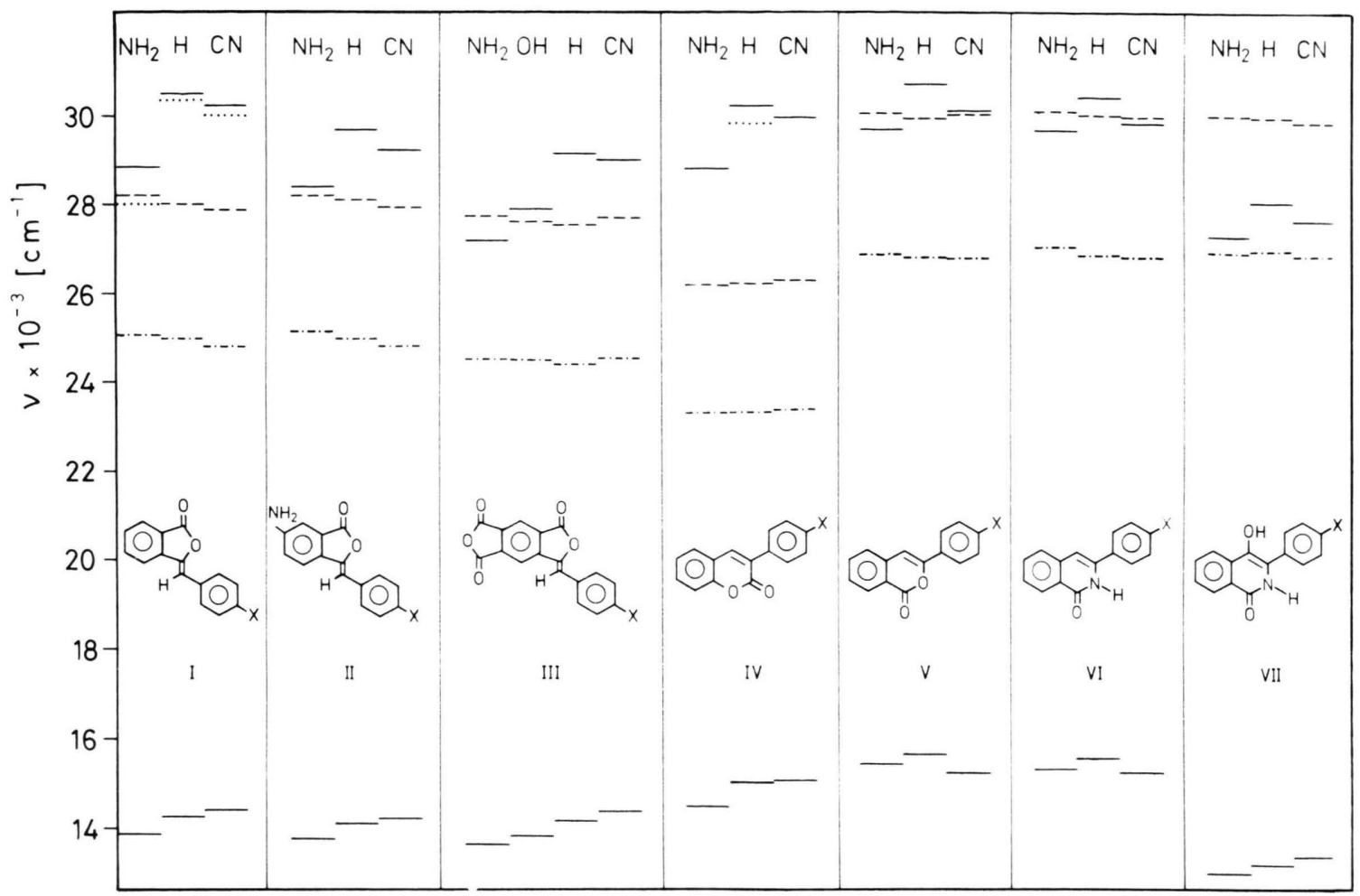

Fig. 1. The ordering of the calculated $T\left(\pi \pi^{*}\right)(-), T\left(\mathrm{n} \pi^{*}\right)(-\cdot-), S\left(\mathrm{n} \pi^{*}\right)(---)$, and $S\left(\pi \pi^{*}\right)(-)$ states of the compounds studied. Dotted line $(\cdots)$ corrected $S\left(\pi \pi^{*}\right)$ states in ethanol (see text).

calculated excitation energies and the experimental spectra is satisfactory, at least for the low lying transitions.

In the discussion of the energy schemes of Fig. 1 we shall concentrate mainly on two features:

(i) the energy gap between the $S\left(\pi \pi^{*}\right)$ and the nonemitting $S\left(\mathrm{n} \pi^{*}\right)$, which in many cases exerts a significant influence on the magnitude of $Q_{\mathrm{f}}$ [6];

(ii) the energy gap between $S\left(\pi \pi^{*}\right)$ and $T\left(\mathrm{n} \pi^{*}\right)$, which allows to estimate the efficiency of intersystem crossing (ISC).

A common feature in all the compounds studied is, that the second singlet $\mathrm{n} \pi^{*}$ state (fourth in III) is separated by about $10000 \mathrm{~cm}^{-1}$ from the $S\left(\pi \pi^{*}\right)$. For the anhydride III three quasi-degenerate lowlying singlet $n \pi^{*}$ states were calculated (see Figure 2). This compound contains three carbonyl groups which are very weakly interacting thus leading to the calculated quasi-degeneracy in the localized $\mathrm{n} \pi^{*}$ excitations. A similar behaviour was encountered in the study of the acenequinones [5]. Nevertheless we will denote also these three states together with the symbol $S\left(\mathrm{n} \pi^{*}\right)$.

When taking into account point (ii) from above, one should bear in mind the El-Sayed rules for ISC [7], and also, that the probability for populating the triplet manifold is larger, if the difference between the zero-point levels of the singlet and triplet states involved is small [8]. As can be seen from Fig. 1 the first triplet states of all compounds studied are of $\pi \pi^{*}$ type and are lying by an amount of $10000 \mathrm{~cm}^{-1}$ below the first singlet states. Therefore, the probability for a $S_{1} \rightarrow T\left(\pi \pi^{*}\right)$ transition is small and it is more likely, that the crossing occurs mainly from $S_{1}$ to the near lying $T\left(\mathrm{n} \pi^{*}\right)$. The second triplet $\mathrm{n} \pi^{*}$ levels in all compounds are in the region $37000-$ $40000 \mathrm{~cm}^{-1}$, i.e. much higher than the first $S\left(\pi \pi^{*}\right)$ states and according to these considerations one could expect mainly $S\left(\pi \pi^{*}\right)$ to $T\left(\mathrm{n} \pi^{*}\right)$ transitions to be effective. 


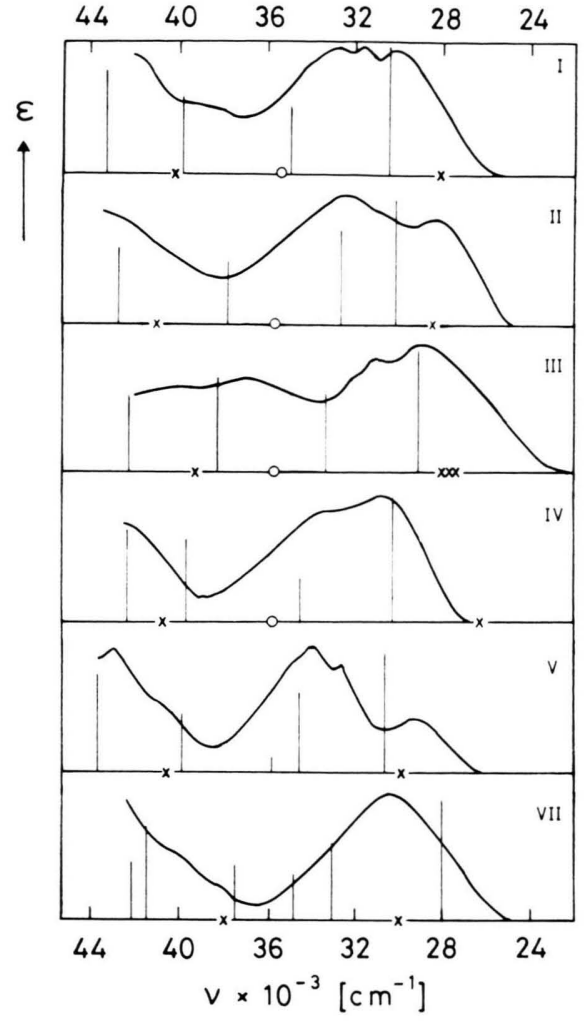

Fig. 2. Comparison of the experimental absorption spectra (in 1,4-dioxane) and the calculated excitation energies for the unsubstituted compounds I, II, III, IV, V, and VII. Vertical lines indicate computed energies and oscillator strengths; $0-$ forbidden $\pi \pi^{*}$ transitions; $\mathrm{x}-$ forbidden $\mathrm{n} \pi^{*}$ transitions. (The spectrum of compound $\mathrm{V}$ was taken from G. Berti, Gazz. Chim. Ital. 86, 655 (1956).)

\subsection{Substituent effects}

$\boldsymbol{S}\left(\pi \pi^{*}\right)-\boldsymbol{S}\left(\mathrm{n} \pi^{*}\right)$. The lowest singlet state in all unsubstituted compounds but VII is of $n \pi^{*}$ type and therefore, one should expect a negligible small fluorescence quantum yield for all these compounds. An acceptor or donor substituent in the $4^{\prime}$ position of the phenyl group causes lowering of the energy of the $S\left(\pi \pi^{*}\right)$ relative to the unsubstituted structure, $\mathrm{X}=\mathrm{H}$. For substituents having Hammett $\sigma$ constants equal in absolute value - as is the case with the $\mathrm{CN}$ and $\mathrm{NH}_{2}$ groups - the effect of the donor substituent is always stronger, as can be derived from the results in Figure 1. However, in concordance with the conclusions in [9], the transition energies of the $S\left(\mathrm{n} \pi^{*}\right)$ states, which are localized on the $\mathrm{C}=\mathrm{O}$ group, remain practically constant. As a result of the narrowing of the $S\left(\mathrm{n} \pi^{*}\right)-S\left(\pi \pi^{*}\right)$ gap one may expect the probability for radiative transitions via $S\left(\pi \pi^{*}\right)$ to increase; in some cases - the $\mathrm{NH}_{2}$ substituted compounds of III, V, and VI - the calculations predict an inversion of $S\left(\mathrm{n} \pi^{*}\right)$ and $S\left(\pi \pi^{*}\right)$ levels, and hence a significant increase in $Q_{\mathrm{f}}$ may be expected. With $1800 \mathrm{~cm}^{-1}$ the effect is most pronounced for III, but even the weaker influence of the $\mathrm{NH}_{2}$ group in compounds $\mathrm{V}$ and VI leads to an inversion of the two levels, since in these cases they lie close to each other in the unsubstituted ones.

The considerable smaller decrease of the excitation energy to the $S\left(\pi \pi^{*}\right)$ level caused by the $\mathrm{CN}$ group leads to an inversion of $S\left(\mathrm{n} \pi^{*}\right)$ and $S\left(\pi \pi^{*}\right)$ only for VI; for V the effect is of the same order and leads to a quasi-degeneracy of the two levels. Consequently, it may be expected, that the donor and acceptor substituted derivatives of type V, VI, and VII will exhibit a significant $Q_{\mathrm{f}}$ even in nonpolar solvents.

The smaller effect of the acceptor substituents in the $\mathrm{X}$ position for all compounds studied is most probably connected with the influence of the $\mathrm{C}=\mathrm{O}$ acceptor group at the other part of the stilbene subchromophore - Figure 1. Considering such a structure as a charge-transfer system of type A-X $(A=$ acceptor $)$ it is natural to expect, that the effect of a donor $\mathrm{X}$ substituent is stronger than with $\mathrm{X}=$ acceptor. These qualitative considerations are borne out by the experimental data for differently substituted BPH's (structure I): $Q_{\mathrm{f}}=0.33, \approx 0.001$, and 0.006 , for $\mathrm{X}=\mathrm{NH}_{2}, \mathrm{H}$, and $\mathrm{CN}$, respectively, in 1,4-dioxane (see Tab. 1; some of the compounds investigated have a poor solubility in cyclohexane and in heptane, at the same time the $Q_{\mathrm{f}}$ 's in non-polar solvents are very low, usually $\leqq 0.001$. For that reason we shall use mainly the experimental data from 1,4-dioxane as a solvent).

$\boldsymbol{S}\left(\pi \pi^{*}\right)-\boldsymbol{T}\left(\mathrm{n} \pi^{*}\right)$. When considering the influence of substituents on the $S\left(\pi \pi^{*}\right)-T\left(\mathrm{n} \pi^{*}\right)$ energy difference, and hence on the ISC between these levels, one should take into account also the absolute value of this difference for $X=H$. For most of the compounds the difference is much larger than any shift of the $S\left(\pi \pi^{*}\right)$ energy caused by substitution. On the other hand, the substituents have practically no influence on the $T\left(\mathrm{n} \pi^{*}\right)$ energy levels. Therefore, it can be expected that introduction of a donor or acceptor substituent will be significant for ISC only for compound VII, where the calculated $S\left(\pi \pi^{*}\right)-$ 
$T\left(\mathrm{n} \pi^{*}\right)$ difference for $\mathrm{X}=\mathrm{H}$ is about $1100 \mathrm{~cm}^{-1}$ and decreases to about $400 \mathrm{~cm}^{-1}$ for $\mathrm{X}=\mathrm{NH}_{2}$ and to $800 \mathrm{~cm}^{-1}$ for $\mathrm{X}=\mathrm{CN}$.

\subsection{Structural effects}

From the data in Fig. 1 we conclude that the excitation energy to the $S\left(\pi \pi^{*}\right)$ level for different structures - I, II, IV, and VI - are close to $30000 \mathrm{~cm}^{-1}$ for $X=H$, which is confirmed also experimentally $[1-3,10]$. A possible explanation for this finding is provided by the pars orbital analysis of the $S_{0} \rightarrow S\left(\pi \pi^{*}\right)$ electronic transitions: in all cases the electronic excitation is localized to a large extent on the stilbene subchromophore with a CT contribution to or from the $\overline{\mathrm{Y}}-\mathrm{C}=\mathrm{O}$ fragment $(\overline{\mathrm{Y}}=\overline{\mathrm{O}}$, $\overline{\mathrm{N}}-\mathrm{H})[10]$.

Next we shall consider the influence of two other structural factors on the energies of the electronic levels - the effect of the $\overline{\mathrm{Y}} \mathrm{C}=\mathrm{O}$ fragment and that of an extra $\mathrm{OH}$ group. The fragment $\overline{\mathrm{Y}}-\mathrm{C}=\mathrm{O}$ consists of the fluorescence-quenching $\mathrm{C}=\mathrm{O}$ group and the donor group $\bar{Y}$, whose presence stimulates emission. Depending on the position of the fragment $\overline{\mathrm{Y}}-\mathrm{C}=\mathrm{O}$ relative to the $\mathrm{C}=\mathrm{C}$ double bond of the stilbene subchromophore we can distinguish two cases:

(i) The $\mathrm{C}=\mathrm{O}$ group is directly connected to the $\mathrm{C}=\mathrm{C}$ bond (compound IV);

(ii) the $\overline{\mathrm{Y}}$ group connects the $\mathrm{C}=\mathrm{O}$ group and the $\mathrm{C}=\mathrm{C}$ bond (compounds I-III, V-VII).

As can be seen from Fig. 1, the $\mathrm{n} \pi^{*}$ excitation energies for the singlet as well as for the triplet state of IV are lower by $2000-4000 \mathrm{~cm}^{-1}$ than those of the other compounds. For $\mathrm{X}=\mathrm{H}$ the $S\left(\pi \pi^{*}\right)$ level of IV lies about $4000 \mathrm{~cm}^{-1}$ above $S\left(\mathrm{n} \pi^{*}\right)$ and the unsubstituted 3-phenylcumarine has fluorescence quantum yields of about 0.001 in 1,4-dioxane and heptane. The large difference between $S\left(\mathrm{n} \pi^{*}\right)$ and $S\left(\pi \pi^{*}\right)$ also suggests a weaker influence of the substituents on $Q_{\mathrm{f}}$ for IV as compared to its isomer V. With more than $6000 \mathrm{~cm}^{-1}$ the $S\left(\pi \pi^{*}\right)-T\left(\mathrm{n} \pi^{*}\right)$ energy difference in IV is almost twice as large as in the other compounds and, therefore, one expects only insignificant ISC. On the other hand, for compounds, in which the $\mathrm{C}=\mathrm{O}$ group is not directly connected to the $\mathrm{C}=\mathrm{C}$ bond of the stilbene subchromophore, one expects higher fluorescence quantum yields; the effects in the two cases (i) and (ii) are opposed.
A comparison of the calculated results for compounds VI and VII allows us to estimate the influence of the additonal $\mathrm{OH}$ group at the $\mathrm{C}=\mathrm{C}$ double bond. The $S\left(\mathrm{n} \pi^{*}\right)$ and $T\left(\mathrm{n} \pi^{*}\right)$ excitation energies are practically not changed by the presence of the $\mathrm{OH}$ group. On the other hand, the energy of the two lowest lying $S\left(\pi \pi^{*}\right)$ and $T\left(\pi \pi^{*}\right)$ states in VII decreases by about $2000 \mathrm{~cm}^{-1}$ and as a result the $S\left(\pi \pi^{*}\right)$ level in VII lies below $S\left(\mathrm{n} \pi^{*}\right)$, irrespective of the substituent $\mathrm{X}$ : for $\mathrm{X}=\mathrm{H}$ by about $2000 \mathrm{~cm}^{-1}$ and for $\mathrm{NH}_{2}$ by $2700 \mathrm{~cm}^{-1}$. Consequently, we conjecture that in the absence of competing factors compounds with structure VII should exhibit appreciable fluorescence quantum yields. But in the case of 1-oxo-3-phenyl-4-hydroxyisoquinoline (VII), as a result of the strong depression of the $S\left(\pi \pi^{*}\right)$ level, the ISC between the $S\left(\pi \pi^{*}\right)$ and $T\left(\mathrm{n} \pi^{*}\right)$ states will probably come into play leading to a diminished fluorescence: for $\mathrm{X}=\mathrm{H}$, the calculated difference between the two states is about $1000 \mathrm{~cm}^{-1}$ and $Q_{\mathrm{f}}=0.1$ in dioxane; for $\mathrm{X}=\mathrm{NH}_{2}$ the energy difference is only about $400 \mathrm{~cm}^{-1}$.

\subsection{Effect of the solvent polarity}

It is a well known effect that $\pi \pi^{*}$ states undergo a bathochromic shift when the solvent polarity increases, whereas $n \pi^{*}$ states are hardly influenced or are shifted to shorter wavelengths. Such a behaviour has also been observed in some of the compounds of the present study (I, II, IV) $[1,3,10]$. For instance, the absorption maxima of the $S\left(\pi \pi^{*}\right)$ state in the $\mathrm{NH}_{2}, \mathrm{H}$, and $\mathrm{CN}$ substituted BPH's are shifted by $1020 \mathrm{~cm}^{-1}, 180 \mathrm{~cm}^{-1}$, and $440 \mathrm{~cm}^{-1}$, respectively, to the red when going from cyclohexane to ethanol [1]. When we take these numbers as a correction to the calculated transition energies we arrive at values for the $S\left(\pi \pi^{*}\right)$ energy levels which are indicated in

Table 1. Fluorescence quantum yields of some substituted benzylidenephtalides (I) in solvents with different polarities [1]. The values measured in 1,4-dioxane are also obtained with the method described in [1].

\begin{tabular}{|c|c|c|c|}
\hline \multirow[t]{2}{*}{$X$} & \multicolumn{3}{|l|}{$Q_{\mathrm{f}}$} \\
\hline & Heptane & 1,4-Dioxane & Ethanol \\
\hline $\mathrm{NH}_{2}$ & 0.01 & 0.33 & 0.54 \\
\hline $\mathrm{OCH}_{3}$ & $\approx 0.001$ & 0.01 & 0.14 \\
\hline $\mathrm{H}$ & $<0.001$ & $\approx 0.001$ & 0.002 \\
\hline $\mathrm{CN}$ & $<0.001$ & 0.006 & 0.12 \\
\hline $\mathrm{NO}_{2}$ & $<0.001$ & 0.008 & 0.12 \\
\hline
\end{tabular}


Fig. 1 as dotted lines. With this correction an inversion between the $S\left(\mathrm{n} \pi^{*}\right)$ and the $S\left(\pi \pi^{*}\right)$ states of the $\mathrm{NH}_{2}$ substituted $\mathrm{BPH}$ arises and one may conclude, that the fluorescence is enhanced by the solvent polarity. The experimental data given in Tab. 1 indicate such an enhancement: the fluorescence quantum yields increase with increasing solvent polarity, for the $\mathrm{NH}_{2}$ substituted $\mathrm{BPH}$ this change amounts to two orders of magnitude. The variation in $Q_{\mathrm{f}}$ for the disubstituted BPH's (II) in solvents with different polarities [3] can be explained in the same way. The small bathochromic shift - about $400 \mathrm{~cm}^{-1}$ - of the longest wavelength absorption maximum for unsubstituted IV in ethanol

[1] P. Nikolov, F. Fratev, and S. Minchev, Z. Naturforsch. 38 a, 200 (1983).

[2] S. Minchev, P. Nikolov, and P. Fratev, Proceedings of the III. Symposium of Photochemistry, May 1982, Potsdam, GDR.

[3] F. Fratev, P. Nikolov, and S. Minchev, Proceedings of the XVI. EUCMOS, September 1983, Sofia, Bulgaria; submitted to J. Mol. Struct.

[4] J. Del Bene and H. H. Jaffe, J. Chem. Phys. 48, 1807, 4050 (1968).

[5] G. Olbrich, O. E. Polansky, and M. Zander, Ber. Bunsenges. Phys. Chem. 81, 692 (1977). vs. dioxane, together with the large energy difference between the $S\left(\mathrm{n} \pi^{*}\right)$ and $S\left(\pi \pi^{*}\right)$ states, is in agreement with the negligible effect on the fluorescence quantum yield, $Q_{\mathrm{f}}$ is about 0.002 in both solvents.

\section{Acknowledgement}

This cooperation was made possible by the Bulgarian Academy of Science in Sofia and the Deutsche Forschungsgemeinschaft in Bonn, the authors express their gratitude to both institutions. We thank Prof. Tyutyulkov and Dr. Hildenbrand for helpful discussions.

[6] J. A. Barltrop and J. D. Coyle, Excited States in Organic Chemistry, Wiley, London 1975.

[7] M. A. El-Sayed, J. Chem. Phys. 38, 2834 (1963).

[8] S. P. McGlynn, T. Azumi, and M. Kinoshita, Molecular Spectroscopy of the Triplet State, Prentice-Hall, Englewood Cliffs 1969.

[9] J. Fabian and H. Hartmann, Light Absorption of Organic Colorants, Springer, Berlin 1980 (Reactivity and Structure, 12).

[10] F. Fratev, V. Enchev, P. Nikolov, and O. E. Polansky, Z. Naturforsch. 39 a, 1143 (1984). 